\title{
El trabajo en equipo desde la mirada de los cuidados y la participación: reflexiones de su aplicación en el Máster en Cooperación al Desarrollo por la UPV
}

\author{
Carola Calabuig Tormo ${ }^{a}$, Álvaro Fernández-Baldor Martínez ${ }^{\text {y }}$ Sergio Belda Miquel \\ a'Departamento Proyectos de Ingeniería, Universitat Politècnica de València, cacator@dpi.upv.es \\ bDepartamento Proyectos de Ingeniería, Universitat Politècnica de València, alferma2@upv.es y Instituto \\ INGENIO UPV-CSIC, Universitat Politècnica de València, serbelmi@ingenio.upv.es
}

\begin{abstract}
The article shows the results obtained in the Master's Degree in Development Cooperation of the Universitat Politècnica de València, related to the application of the participation and care approach in teamwork competence, from the end of the 2016-2017 academic year, to current 2017-2018 academic year. The work collects what has been carried out since the 2015-2016 academic year, allowing to assess the improvements introduced and analyzing the evolution in two different groups of students. The most outstanding innovation in the last year is the improvement of the rubric for the evaluation of the competence, with the assistance of a Valencian DNGO in the assessment. There has also been a transfer of the competence to the space of external curricular internships of the master. The results continue to show the need to accompany the process, as well as to reinforce the understanding and application of some dimensions of teamwork from the adopted approaches, in order to consolidate the proposal.
\end{abstract}

Keywords: teamwork, competences, care approach, assesment

\begin{abstract}
Resumen
La comunicación muestra los resultados obtenidos en el Máster en Cooperación al Desarrollo por la Universitat Politècnica de València, relativos a la aplicación del enfoque de cuidados y la participación a la competencia transversal trabajo en equipo, desde finales del curso 2016-2017, hasta lo que lleva de avance el curso 2017-2018. El trabajo recoge lo llevado a cabo desde el curso 2015-2016, permitiendo valorar las mejoras introducidas y analizando la evolución en dos grupos de alumnado diferente. La innovación más destacada en el último año es la mejora de la rúbrica para la evaluación de la competencia, con el concurso de una ONGD valenciana en el asesoramiento. También ha habido un traslado de la competencia al espacio de las prácticas externas curriculares del máster. Los resultados siguen mostrando la necesidad de acompañar el proceso, así como reforzar el entendimiento y aplicación de algunas dimensiones del trabajo en equipo desde los enfoques adoptados, para consolidar la propuesta.
\end{abstract}

Palabras clave: trabajo en equipo, competencias transversales, enfoque de cuidados, evaluación

\section{Introducción}

Este artículo aborda el trabajo realizado en el último año por el profesorado del Máster Universitario en Cooperación al Desarrollo (MCAD) por la Universitat Politècnica de València 
El trabajo en equipo desde la mirada de los cuidados y la participación: reflexiones de su aplicación en el Máster en Cooperación al Desarrollo por la UPV

(UPV), por introducir el enfoque de cuidados y la participación en la competencia transversal trabajo en equipo. Recogiendo para ello los antecedentes de dicha experiencia.

En los estudios relacionados con la cooperación al desarrollo existe un intenso debate sobre el papel que debe desempeñar la formación de profesionales del desarrollo. Existe una corriente hegemónica, dominada por una perspectiva instrumental y poco crítica con la gestión del desarrollo (Gulrajani, 2010; Mosse, 2005), según la cual las personas dedicadas a la gestión del desarrollo deben ser "buenos técnicos", capaces de manejar los instrumentos de gestión adecuados para identificar las mejores soluciones y guiar adecuadamente el cambio en la dirección deseada (Johnson y Thomas, 2007). Como Peris et al. (2013) mencionan, esta perspectiva implicaría que los procesos formativos en gestión de proyectos de desarrollo se centren en proveer a las y los estudiantes capacidades para manejar herramientas que permitan alcanzar de manera eficiente objetivos prefijados de desarrollo mediante una secuencia lineal de pasos, o al menos mediante una planificación estricta (Clarke and Oswald, 2010).

Sin embargo, otra corriente más crítica consideraría que las personas gestoras del desarrollo deberían poner en el centro la naturaleza siempre compleja y política de los procesos de desarrollo, en los que las relaciones de poder juegan un lugar fundamental (McCourt y Gulrajani, 2010; Eyben et al, 2015). Esta visión entendería que los programas de educación superior en gestión del desarrollo deberían focalizarse en generar competencias para entender, desvelar y moverse en contextos complejos y embebidos en relaciones de poder, desde una perspectiva, abierta, flexible, colaborativa y adaptativa. Entre estas competencias, tiene un rol fundamental el trabajo en equipo, la participación y la actitud cooperativa para un trabajo productivo, respetuoso y transformador. Distintos autores del ámbito del desarrollo han apuntado que existen numerosos métodos y aproximaciones pedagógicas para desarrollar capacidades que vayan más allá del gerencialismo en la práctica del desarrollo (ver ejemplos específicos en Ortiz Aragon, 2010; Pearson, 2010; Pettit, 2006; Soal, 2010), y que van desde el aprendizaje experiencial a métodos creativos que apelan a las emociones y el uso del cuerpo.

Es bajo esta última perspectiva que se alinea el MCAD, intentando replantear las metodologías de enseñanza-aprendizaje actualmente empleadas en la gestión del desarrollo y apostando por una educación transformadora, que genere la participación activa y comprometida de todas las personas a favor de la justicia, la equidad de género y la sostenibilidad social y ambiental. Para llevar a cabo esta misión se apuesta por incorporar en la docencia diversos enfoques como el de género, el de cuidados, la participación o la sostenibilidad.

El MCAD es un título oficial de la UPV de 90 ECTS, orientado a formar profesionales de la gestión del desarrollo con competencias que les permitan una práctica crítica de la cooperación. El MCAD se desarrolla en el entorno de la UPV, una universidad técnica con una perspectiva aún muy instrumental, racionalista y limitada de las capacidades que una universidad debe desarrollar en su alumnado; muy orientada a la empleabilidad, y con un uso aún muy extendido de prácticas de enseñanza-aprendizaje poco activas. Sin embargo, la UPV ha ido generando también estrategias y espacios alternativos, para tratar de que su profesorado genere y proponga una perspectiva más amplia de la enseñanza superior y de los procesos de enseñanzaaprendizaje. Por ejemplo, está tratando de impulsar progresivamente el trabajo en trece 
competencias transversales en todas las titulaciones y asignaturas, así como en el uso de metodologías activas de enseñanza-aprendizaje para desarrollarlas.

En este artículo mostramos el trabajo realizado por el profesorado del equipo de innovación educativa (EICE) del MCAD, denominado EICE-MACODE, por incorporar el enfoque de cuidados y la participación en la competencia transversal trabajo en equipo. Se amplía de esta forma el trabajo previo realizado, disponiéndose de una contextualización más detallada de los enfoques adoptados y de la innovación, así como de los primeros avances, en Calabuig, Fernández-Baldor y Belda (2017). El trabajo realizado se enmarca en dos Proyectos de Innovación y Mejora Educativa (PIME) emprendidos desde el EICE-MACODE, uno de ellos en activo, apoyados desde el Instituto de Ciencias de la Educación y el Vicerrectorado de Estudios, Calidad y Acreditación de la UPV.

El enfoque de cuidados pone en evidencia que nuestro actual modelo de desarrollo, centrado en los mercados, es un modelo injusto, insostenible y generador de múltiples desigualdades, y en el que los trabajos de cuidados, imprescindibles para el sostenimiento de la vida, son casi invisibles, poco reconocidos, mal pagados, muchas veces relegados a que los realicen terceras personas (casi siempre las mujeres) y que tienen un desigual reparto e impacto entre hombres y mujeres (InteRed, 2017). Por ello, desde la mirada de cuidados se reivindica la posición clave de éstos en cualquier proceso de desarrollo, revalorizando los aportes que nacen precisamente del cuidado de la vida, frente a lo productivo, competitivo y excluyente de los modelos predominantes (Herrero, 2013). Así, planteamos incorporar esta mirada en el trabajo en equipo que desarrolla el alumnado del MCAD de manera que nos permita mirar la realidad teniendo en cuenta el cuidado del planeta donde vivimos (tanto a las otras personas como a la naturaleza), el cuidado en el hogar y nuestro propio cuidado.

\section{Objetivos}

La presente comunicación recoge el trabajo realizado entre abril de 2017 (curso académico 2016-2017) y el curso 2017-2018 (hasta marzo de 2018 inclusive) para mejorar y evaluar el desarrollo en el alumnado del MCAD de la competencia transversal trabajo en equipo, en tres asignaturas del primer curso del MCAD, desde el enfoque de los cuidados y la participación, siendo los objetivos al poner en marcha la iniciativa los siguientes:

- Integrar en la caracterización y variables definitorias de la competencia trabajo en equipo los enfoques de participación y de cuidados mediante la colaboración entre la UPV y la ONGD española InteRed.

- Llevar a cabo formación específica para el alumnado sobre la competencia trabajo en equipo desde los enfoques adoptados.

- Incorporar mecanismos suficientes para implementar la competencia de trabajo en equipo, desde la participación y cuidados, en tres de las cinco asignaturas del máster.

- Poner en práctica y contrastar herramientas para la adecuada evaluación cualitativa y/o cuantitativa de dicha competencia, tanto en espacios formales como informales de aprendizaje, en el seno del máster. 
El trabajo en equipo desde la mirada de los cuidados y la participación: reflexiones de su aplicación en el Máster en Cooperación al Desarrollo por la UPV

- Potenciar entre el alumnado la capacidad de reflexión sobre el proceso seguido en el trabajo en equipo, tanto el llevado a cabo de forma presencial en el aula, como fuera de ella (prácticas externas curriculares).

\section{Desarrollo de la innovación}

El propósito de la innovación educativa ha sido consolidar y mejorar el trabajo que se ha venido realizando hasta la fecha sobre la competencia trabajo en equipo en el Máster en Cooperación al Desarrollo (MCAD) por la UPV. Para ello, hemos incorporado de manera más profunda los enfoques de participación y de cuidados para trabajar la mencionada competencia, a partir de la experiencia desarrollada durante los cursos 2015-2016 y 2016-2017. A lo largo del trabajo, para la competencia será empleada la denominación Trabajo en equipo y liderazgo (código CT06), ya que está así establecida en la UPV; sin embargo, la dimensión liderazgo no ha sido abordada desde el máster hasta la fecha con la misma profundidad.

\subsection{El trabajo en equipo desde la participación y los cuidados}

Aunque la competencia transversal de trabajo en equipo es ampliamente abordada en muchos planes de estudio, proyectos y equipos de innovación educativa, aquí la particularidad ha sido que el trabajo en equipo fuera de corte colaborativo. Esta preocupación ha estado presente con fuerza desde el inicio de la titulación. La misma busca contribuir a la formación de profesionales del campo del desarrollo y la cooperación, como ciudadanas/os globales que sean capaces de ejercer su agencia individual y colectiva para el logro de modelos alternativos de mayor justicia social. Se trata, además, de que el alumnado del Máster sea capaz de trabajar en equipo en contextos de gran complejidad e incertidumbre y en los que las relaciones de poder juegan un papel fundamental (McCourt y Gulrajani, 2010; Eyben et al, 2015). Todo ello es habitual en los procesos de desarrollo. El propio alumnado que ha realizado sus prácticas externas internacionales así lo ha experimentado a pequeña escala.

Esta inquietud ha adquirido, si cabe, más fuerza durante los últimos dos cursos académicos, constatándose que debían ponerse más medios en el máster para trabajar y evaluar el trabajo en equipo como competencia indispensable en este área. Y a hacerlo desde una perspectiva más crítica, contraponiéndose a perspectivas tecnocráticas y gerenciales (se considera que éstas no contribuyen a abordar las causas estructurales de la pobreza y la exclusión sino que, al contrario, refuerzan las relaciones desiguales de poder).

Así mismo, el alumnado del máster, a través de los Comités de Seguimiento de las asignaturas, había expresado durante varios cursos la necesidad de: (i) disponer de más herramientas para entender las dimensiones del trabajo en equipo colaborativo; (ii) a poder ser acompañadas en ese proceso de aprendizaje en la práctica; (iii) y a poder evaluarlo. El EICE-MACODE decide apostar durante el curso 2016-2017, de manera progresiva y con diferentes grados de intensidad, por varias competencias transversales, principalmente CT06 Trabajo en equipo y liderazgo. Se adoptaron entonces dos enfoques específicos: el enfoque participativo y el enfoque de cuidados, combinando la experiencia y esfuerzo del personal docente del Máster con el apoyo de profesionales externas. Por un lado, el enfoque de participación ha sido esencial en la definición del enfoque pedagógico general y en la metodología de enseñanza-aprendizaje del MCAD (ver Peris et al., 2013; Belda et al., 2016),

(cc) EY-NC-ND 2018, Universitat Politècnica de València

Congreso IN-RED (2018) 
no solo porque se enseñan diferentes metodologías y técnicas participativas, sino porque el alumnado tiene que aplicarlas en sus trabajos en equipo: desde debates y discusiones grupales, juegos de rol o teatro, hasta la realización de IAP (investigación-acción participativa), ApS (Aprendizaje-Servicio) o Aprendizaje en la Acción. Por otro lado, se ha trabajado desde el denominado enfoque de cuidados, que algunas ONGD como InteRed están implementando desde la llamada pedagogía de cuidados. Como se avanzó en la introducción, este enfoque pone en el centro una combinación de la ética de la justicia y la ética del cuidado, centrándose: (i) en el reconocimiento del derecho individual y colectivo al cuidado (Carrasco, Borderías y Torns, 2011); (ii) en la dimensión política de lo personal; (iii) y en la dimensión ecológica del cuidado. Esto implica poner en el centro la igualdad dentro de las relaciones personales, desmontando jerarquías y privilegios, así como la atención de las necesidades que todas las personas pueden tener. El reconocimiento del otro/a como sujeto de derecho al cuidado lleva además a atender a la singularidad de cada persona o comunidad y a tener que dar espacio a la diversidad para que la diferencia no se convierta en desigualdad (De Blas, 2016). En general, un enfoque de cuidados supone implicarse en el bienestar de las demás personas desde posturas ecofeministas (Herrero, 2013). Supone ser conscientes de que el bienestar es una experiencia personal, vinculada a los intereses y deseos personales, a la razón, al cuerpo y a las emociones.

La incorporación de ambos enfoques, como se expondrá, ha derivado en un replanteamiento de las dimensiones que el trabajo en equipo debe tener y que debería fomentarse desde el máster.

\subsection{Insumos recibidos del curso 2016-2017}

Si bien las diversas asignaturas del máster seguirían siendo punto de control de otras competencias, se toma la decisión de profundizar en la de trabajo en equipo: garantizando una mejor coordinación entre el profesorado; buscando una mayor coherencia entre métodos empleados en las diferentes asignaturas; incorporando las demandas del alumnado fruto de coevaluaciones realizadas en los comités de seguimiento de las asignaturas (por ejemplo: precisar las rúbricas, dar más herramientas para el trabajo en equipo o dimensionar mejor el trabajo en equipo autónomo); revisando el sistema de rúbricas para la evaluación; e implicando a las ONG de Desarrollo que forman parte del Consejo Asesor del máster.

Es por ello que, durante el curso 2016-2017, se redefinieron unidades temáticas de algunas asignaturas y se realizó el esfuerzo de revisar internamente los métodos docentes y de evaluación. Se buscaba que el trabajo en equipo fuera puesto en práctica con el rigor y profundidad requeridos, así como de forma coherente con los planteamientos del máster expuestos con anterioridad. En Calabuig, Fernández-Baldor y Belda (2017) se dispone de una descripción pormenorizada de las actividades realizadas en el curso 2016-2017 y de los primeros resultados obtenidos hasta marzo de 2017. De forma resumida, se llevaron a cabo las siguientes: (a) dos talleres sobre trabajo en equipo, uno en cada cuatrimestre, específicamente diseñados para el alumnado de primer año del máster e impartido por personal especializado de la ONGD InteRed; (b) redefinición del sistema de evaluación de la competencia transversal, incorporando más espacios y actividades secuenciadas y simplificando el cuestionario; (c) respecto a los cuestionarios de autoevaluación y evaluación por pares, se decide reducir a cuatro los criterios de evaluación y no emplear un sistema de rúbricas como tal, sino aplicar una escala cualitativa de valoración (cuatrimestre A)

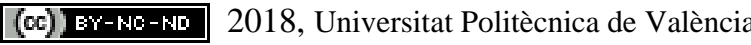

Congreso In-Red (2018) 
El trabajo en equipo desde la mirada de los cuidados y la participación: reflexiones de su aplicación en el Máster en Cooperación al Desarrollo por la UPV

y otra cuantitativa (cuatrimestre B); (d) evaluación de los mecanismos puestos en marcha al final del cuatrimestre A, tanto en el seno del EICE-MACODE, como con el alumnado del máster (en aula y a través de los Comités de Seguimiento de asignaturas).

\begin{tabular}{|c|c|c|c|c|c|}
\hline \multicolumn{6}{|c|}{ Valoración de la competencia trabajo en equipo y liderazgo } \\
\hline \multirow{2}{*}{ Criterio } & \multirow{2}{*}{ Qué comprende el criterio } & \multicolumn{4}{|c|}{ Descriptores } \\
\hline & & A & $\mathrm{B}$ & C & $\mathrm{D}$ \\
\hline $\begin{array}{l}\text { Escucha activa, } \\
\text { empatía, cuidados } \\
\text { y respeto }\end{array}$ & $\begin{array}{l}\text { En los espacios de trabajo se muestra una persona con interés. } \\
\text { Focaliza la atención en la dinámica de grupo, sin desviarse a } \\
\text { otros asuntos. Diferencia lo que son espacios de comunicación } \\
\text { relativos al trabajo del máster de los espacios privados. Da su } \\
\text { opinión sin monopolizar las conversaciones. El lenguaje } \\
\text { empleado en las intervenciones en general es inclusivo y } \\
\text { respetuoso. Es capaz de empatizar con l@s compañer@s y } \\
\text { actúa desde la cultura de los cuidados, no buscando solamente } \\
\text { un enfoque "eficientista" de la tarea. }\end{array}$ & & & & \\
\hline $\begin{array}{l}\text { Gestión de } \\
\text { conflictos }\end{array}$ & $\begin{array}{l}\text { Tiene en cuenta diferencias culturales o de otro tipo que } \\
\text { pueden dificultar un adecuado entendimiento y provocar } \\
\text { malestar. Busca integrar a todas las personas que forman parte } \\
\text { del equipo, incorporándolas a la conversación o a la sesión } \\
\text { concreta. Es capaz de aclarar su punto de vista de manera } \\
\text { adecuada frente a sus compañeras, así como reaccionar bien } \\
\text { ante visiones contrapuestas. Es capaz de aportar soluciones a } \\
\text { posibles conflictos generados entre los miembros del equipo }\end{array}$ & & & & \\
\hline Liderazgo social & $\begin{array}{l}\text { Sus intervenciones invitan a otros/as a profundizar en } \\
\text { cuestiones particulares. Valora, recoge e integra ideas } \\
\text { formuladas por sus compañeros/as para profundizar en ellas. } \\
\text { Motiva a las compañeras, promueve la distribución de roles y } \\
\text { la cohesión del grupo. }\end{array}$ & & & & \\
\hline $\begin{array}{l}\text { Responsabilidad, } \\
\text { compromiso y } \\
\text { rendición de } \\
\text { cuentas }\end{array}$ & $\begin{array}{l}\text { Se muestra como una persona proactiva en el trabajo en equipo } \\
\text { y comprometida con los objetivos a conseguir. Es responsable } \\
\text { con las tareas encomendadas y da cuentas de las mismas, } \\
\text { comunicando avances, resultados, etc., al resto del equipo. Es } \\
\text { puntual para el inicio de las reuniones. }\end{array}$ & & & & \\
\hline \multicolumn{6}{|c|}{$\begin{array}{l}\text { Descriptores cualitativos: } \\
\text { A: Ocurre casi siempre / Se da de forma ejemplar } \\
\text { B: Ocurre con frecuencia/Se da de forma adecuada } \\
\text { C: Ocurre con poca frecuencia/ Se da de forma mejorable } \\
\text { D: No ocurre casi nunca/Se da de forma claramente insatisfactoria }\end{array}$} \\
\hline
\end{tabular}

Figura 1: Tabla tipo para la valoración de la competencia trabajo en equipo durante el curso 2016-2017 Fuente: elaboración propia

La figura 1 muestra la tabla que se utilizó durante el curso 2016-2017 para evaluar la competencia. Como veremos en la siguiente sección, esta ha sufrido importantes modificaciones en el curso 2017-2018.

\subsection{Actividades realizadas en el curso 2017-2018}

Las principales actividades desarrolladas en el marco del proyecto en el presente curso académico 2017-2018, fruto de la evaluación del trabajo realizado en el curso anterior y hasta la fecha, han sido:

- Establecimiento de una mayor colaboración entre el EICE-MACODE y la ONGD InteRed, ONGD española de referencia en materia de educación para el desarrollo y educación para la ciudadanía global y que ha desarrollado una aportación propia denominada ética revolucionaria de los cuidados (InteRed, 2014).

- Taller específico para el alumnado de primer año sobre trabajo en equipo y liderazgo con enfoque de cuidados y participación (octubre de 2017), con la consiguiente generación de materiales revisados desde los enfoques adoptados. 
- Incorporación de lecciones aprendidas durante 2016-2017 a los mecanismos de trabajo y acompañamiento en el desarrollo de la competencia aplicada a tres de las asignaturas del máster (primer curso de docencia). Uno de los elementos identificados a mejorar fue el diseño de la rúbrica para la evaluación, así como los espacios para el seguimiento.

- Rediseño del sistema de evaluación de la competencia CT06 en tres de las asignaturas del máster, mediante adecuación de las rúbricas según los enfoques adoptados y a partir de diversos formatos de rúbricas aconsejados por el Instituto de Ciencias de la Educación (ICE) de la UPV. En efecto, a fin de mejorar la incorporación de los enfoques de participación y cuidados, y siguiendo las pautas del ICE, durante este curso académico se ha realizado un gran esfuerzo por rediseñar la rúbrica (ver tablas 1 y 2). En su definición han participado: el profesorado del EICE-MACODE, la ONGD InteRed y el alumnado del presente curso. La nueva rúbrica contiene cinco dimensiones evaluativas: participación; responsabilidad compartida y compromiso; escucha activa; empatía y comprensión mutua; gestión de conflictos. A su vez, cada dimensión se divide en diferentes indicadores, hasta un total de 17. El contenido y significado de cada uno de los indicadores, adaptado a la rúbrica, es lo que más trabajo ha generado al profesorado, al alumnado y a la ONGD. Para el cuatrimestre A (primer momento en el empleo de la rúbrica, más de aproximación) se ha adoptado una escala cualitativa (A,B,C y D) para los descriptores. Para el cuatrimestre B, los descriptores se han medido mediante puntuación, permitiendo de esta forma profundizar en el análisis cuantitativo.

- Generación de espacios en el aula con el alumnado, de forma planificada, para la reflexión individual y colectiva respecto al desempeño de la competencia.

- Medición del desempeño respecto a esta competencia, a estudiantes que realicen en 20172018 sus prácticas externas (segundo año del máster). Se ha llevado a cabo incorporando preguntas específicas en el guión que deben emplear para elaborar su memoria de prácticas una vez las finalizan, y a la fecha se están realizando entrevistas a dicho alumnado.

La tabla 1 muestra las dimensiones, e indicadores para cada una de ellas, que se han definido para caracterizar la competencia, enfatizando la participación y los cuidados. Para cada indicador se ha desarrollado una rúbrica, que se ha entregado al alumnado para que pudiera realizar su autoevaluación y la coevaluación. Se trata de la empleada en el cuatrimestre A, especificando más el contenido de algunos descriptores y haciendo uso de escala cuantitativa, como se mencionó anteriormente. El alumnado ha participado en este proceso de definición, aportando sus opiniones.

Debido a las limitaciones de espacio y por la imposibilidad de incorporar de forma legible toda la información, no es posible mostrar todas las rúbricas definidas, por lo que se han incluido únicamente las relativas a las dimensiones empatía y comprensión mutua y escucha activa. La tabla 2 muestra descriptores cuantitativos (se decide que una A empleada en el primer cuatrimestre pase a ser en la rúbrica para el segundo semestre una escala de 9 a 10; una B, escala de 7 a 8 ; una C, escala de 5 a 6 y una D, escala de 0 a 4). 
El trabajo en equipo desde la mirada de los cuidados y la participación: reflexiones de su aplicación en el Máster en Cooperación al Desarrollo por la UPV

Tabla 1. Dimensiones e indicadores de la competencia trabajo en equipo

\begin{tabular}{|c|c|}
\hline DIMENSIÓN & INDICADORES \\
\hline \multirow{4}{*}{$\begin{array}{l}\text { Participación: Participar y colaborar } \\
\text { activamente en las tareas del equipo, } \\
\text { fomentando la confianza y cordialidad } \\
\text { y la orientación al trabajo conjunto }\end{array}$} & Ideas propias y voz \\
\hline & Colaboración y contribución al trabajo en equipo. \\
\hline & Participación activa en la toma de decisiones \\
\hline & Respeto a las diferencias \\
\hline \multirow{4}{*}{$\begin{array}{l}\text { Responsabilidad compartida y } \\
\text { compromiso }\end{array}$} & Puntualidad \\
\hline & $\begin{array}{l}\text { Colaboración en la definición, asignación y realización de } \\
\text { las tareas }\end{array}$ \\
\hline & Corresponsabilidad y complementariedad \\
\hline & Búsqueda del bien común. Actuaciones desde lo colectivo \\
\hline \multirow{2}{*}{$\begin{array}{l}\text { Escucha Activa: Escuchar no solo lo } \\
\text { que la persona expresa directamente, } \\
\text { sino entender también sus sentimientos, } \\
\text { ideas o emociones que subyacen a lo } \\
\text { que está diciendo }\end{array}$} & $\begin{array}{l}\text { Concentración y atención al mensaje. Asertividad a la hora } \\
\text { de escuchar }\end{array}$ \\
\hline & Respeto \\
\hline \multirow{5}{*}{$\begin{array}{l}\text { Empatía y comprensión mutual: La } \\
\text { empatía es la base para una escucha } \\
\text { activa y supone ponerse en el lugar del } \\
\text { otro/a, calzar sus zapatos: ser capaz de } \\
\text { entenderle y comprenderle desde "su } \\
\text { escala de importancia”" }\end{array}$} & Reconocimiento del trabajo \\
\hline & $\begin{array}{l}\text { Comprensión de las circunstancias personales, capacidad de } \\
\text { empatía }\end{array}$ \\
\hline & Solidaridad \\
\hline & Actitud positiva \\
\hline & Práctica de los cuidados \\
\hline \multirow{2}{*}{ Gestión de conflictos } & Conciliación y mediación \\
\hline & Ambiente pacífico basado en el diálogo \\
\hline
\end{tabular}

Fuente: elaboración propia

\section{Resultados}

Se recogen a continuación los principales resultados obtenidos. Por limitaciones de espacio, se centra el análisis en aquellos más significativos relativos al curso 2017-2018, tanto en estudiantes de primer año (se trata de un grupo de 24 personas), como en aquellos que durante el presente curso han realizado sus prácticas externas curriculares (se ha podido disponer de información de 9 estudiantes que ya han regresado de sus destinos). Se han tratado en el apartado 4.1, mientras que otros resultados se han resumido en el apartado 4.2.

\subsection{Resultados relativos al desempeño de la competencia}

En cuanto al cuatimestre A, algunas reflexiones llevadas a cabo por el alumnado durante los espacios ofrecidos para la reflexión individual y grupal dan muestra de que, con acompañamiento y herramientas, son capaces de darle un sentido diferente al trabajo en equipo: "pese a nuestras diferencias, creo que las asumimos respetuosa y empáticamente y conseguiremos sacarlo adelante y con buena calidad" (estudiante 1); "para mí, que un trabajo sea un buen trabajo en equipo, cada uno debería tener la capacidad de reconocer y valorar lo que cada uno aporta" (estud. 2); "lo que más me ha sorprendido es poder escuchar de manera más activa, construyendo mis ideas junto con las ideas que el grupo aporta” (estud. 3). 
Tabla 2. Rúbricas para la valoración de la competencia trabajo en equipo durante el curso 2017-2018

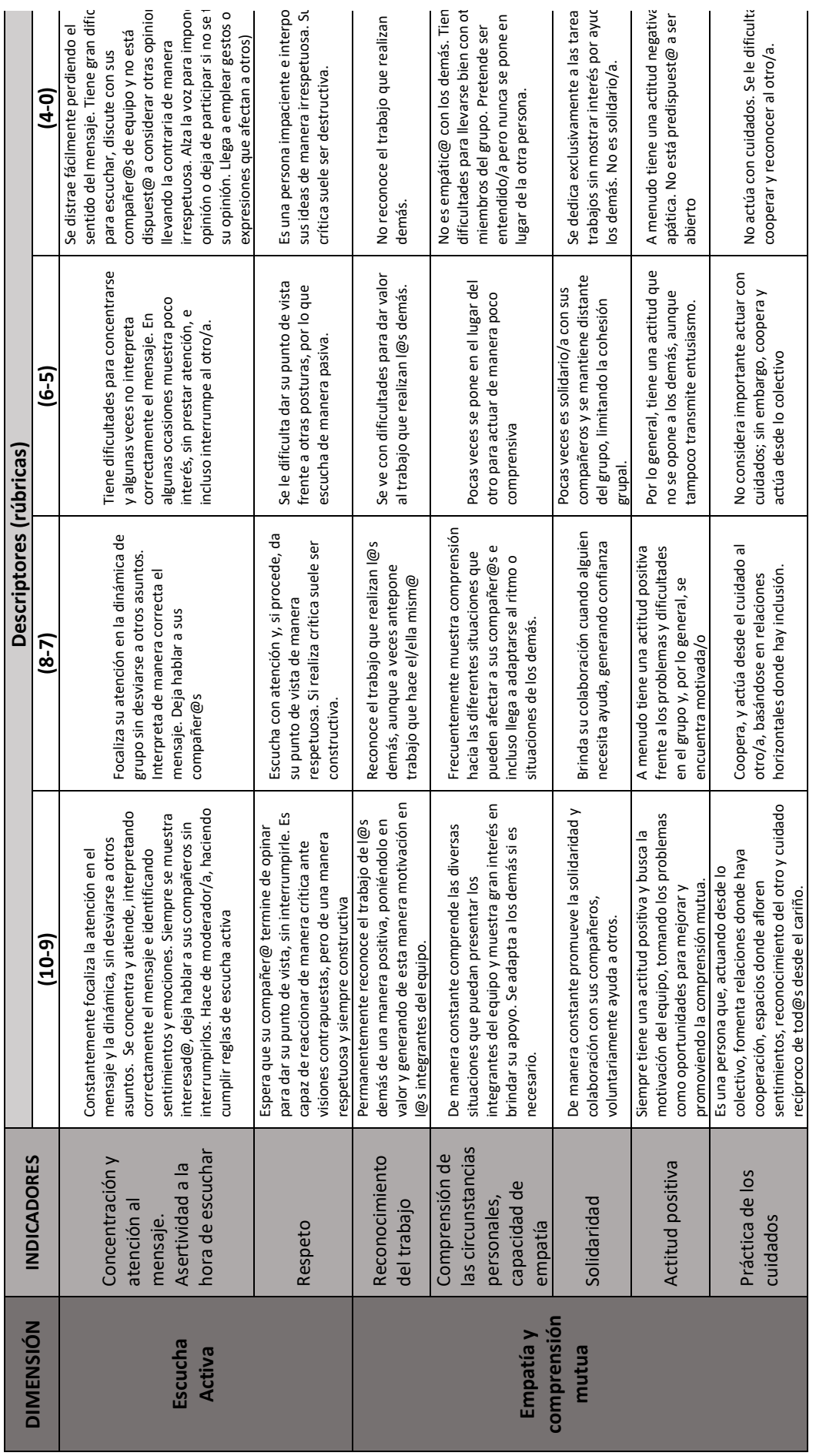

Fuente: elaboración propia

(cc)) EY-Nc-ND 2018, Universitat Politècnica de València Congreso In-Red (2018) 
El trabajo en equipo desde la mirada de los cuidados y la participación: reflexiones de su aplicación en el Máster en Cooperación al Desarrollo por la UPV

En cuanto al desempeño propiamente dicho de la competencia, ha habido diversidad de resultados, sobre una base de 6 equipos de trabajo y un total de 23 cuestionarios (sobre 24 estudiantes) de auto-evaluación y co-evaluación respondidos, relativos a la asignatura Cooperación al Desarrollo (el trabajo en equipo contribuye en un $40 \%$ a la nota final). Por un lado, ha habido equipos que han funcionado de forma muy satisfactoria, donde dimensiones básicas del trabajo en equipo como responsabilidad en la asignación de las tareas o contribución al trabajo en equipo han favorecido el buen clima y la presencia de otras dimensiones más relacionadas con el cuidado, como la comprensión mutua. Sin embargo, en otros casos, ha habido equipos que no han funcionado adecuadamente en los que el conflicto, incluso a pesar de haber sido abordado en la reunión intermedia de seguimiento, no ha sido completamente resuelto. En estos casos, se aprecia cómo las valoraciones por pares (co-evaluación) calificadas como C o D se han otorgado en indicadores como asertividad, búsqueda del bien común, comprensión de las circunstancias personales, práctica de los cuidados o solidaridad. El anonimato en el cuestionario ha favorecido la sinceridad y, aunque las valoraciones eran cualitativas (A, B, C y D), se aprecia cómo las mayores discrepancias entre lo que el/la estudiante valora de sí mismo y cñomo es valorado por sus compañeros/as se dan en los equipos en los que ha aflorado algún tipo de conflicto. También algunas estudiantes han expresado la importancia que ha tenido esta forma de aproximarse y practicar el trabajo en equipo: "Creo que este tipo de evaluacion por un lado, es importante y rompe con los escquemas tradicionales que existen en las Universidades y en diferentes lugares de trabajo, en cuanto toma en consideracion la persona con sus esencias y sus valores y no solo con lo que "produce" (estudiante 4).

En el cuatimestre B, en la Unidad Temática 1 de la asignatura Gestión del Ciclo del Proyecto (que supone prácticamente la mitad de los créditos del total de la asignatura), todo el trabajo se realiza en equipo. Además de la nota del propio trabajo, que se evalua por grupos, existe un 5\% de la nota final de la asignatura que consiste en autoevaluación y coevaluación de cada miembro del equipo según la rúbrica de evaluación mostrada en el apartado anterior. Para la evaluación, el profesor responsable de la asignatura dejó unos 15 minutos para que individualmente el alumnado leyera la rúbrica. Posteriormente, se dedicaron 30 minutos de reflexión para cada grupo, para que pudieran comentar cómo había sido la dinámica del trabajo en equipo y cómo se habían sentido. En definitiva, se trataba de comentar grupalmente cómo habían trabajado en equipos según la rúbrica. A continuación, el profesor dejó unos 30 minutos para que individualmente se autoevaluaran y evaluaran a cada uno de sus compañeros y compañeras de grupo.

De las 23 personas que cursan el máster, disponemos de 22 respuestas, pues una de las personas que ese día no asistió a clase todavía no ha entregado sus valoraciones.

La figura 2 muestra una gráfica que compara los resultados generados por el alumnado en lo que se refiere a su autoevaluación y la coevaluación. La figura muestra cómo la puntuación que se otorgan individualmente (autoevaluación), es similar a la que le otorga su grupo (coevaluación). Las principales excepciones las conforman el alumno $\mathrm{n}^{\circ} 8$, quien es muy crítico con su desempeño en la competencia (alrededor de 4 puntos frente a los casi 7,5 que le otorga su grupo)

(cc) EY-NC-ND 2018, Universitat Politècnica de València

Congreso IN-RED (2018) 
y el alumno 17, quien contrariamente se autoevalúa con prácticamente 2 puntos más que los que le otorga su equipo.

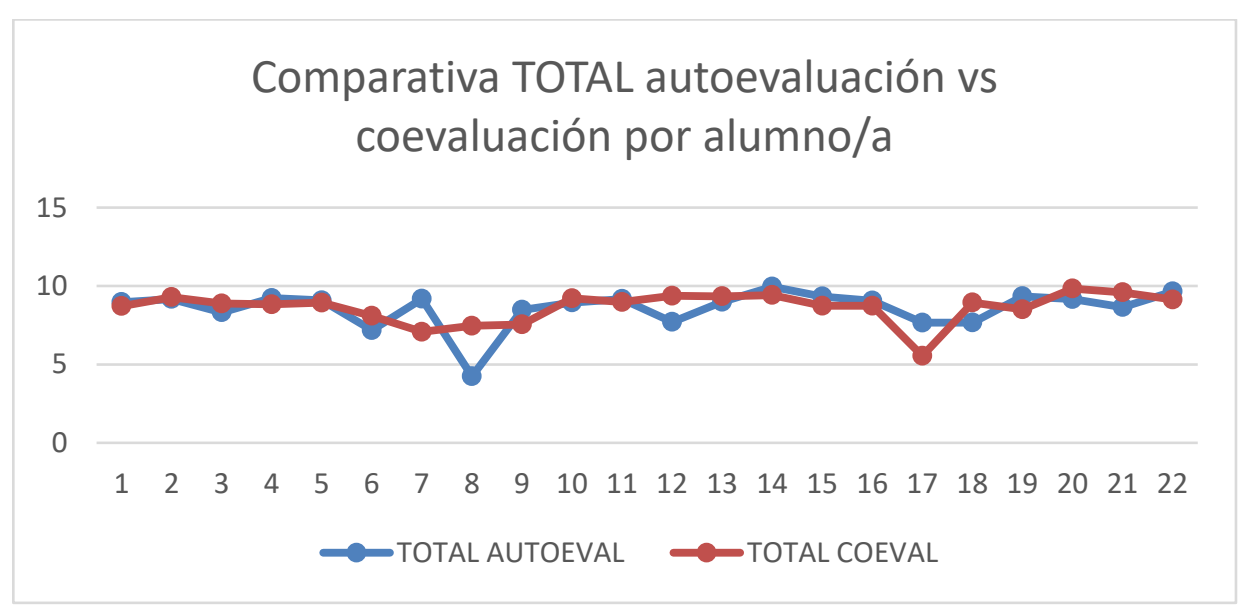

Figura 2: Comparativa entre la autoevaluación y la coevaluación para los 22 alumnos de la muestra Fuente: elaboración propia

Dejando las particularidades especificas del alumnado, y hablando de cifras medias, en la figura 4 podemos observar la puntuación por cada una de las cinco dimensiones.

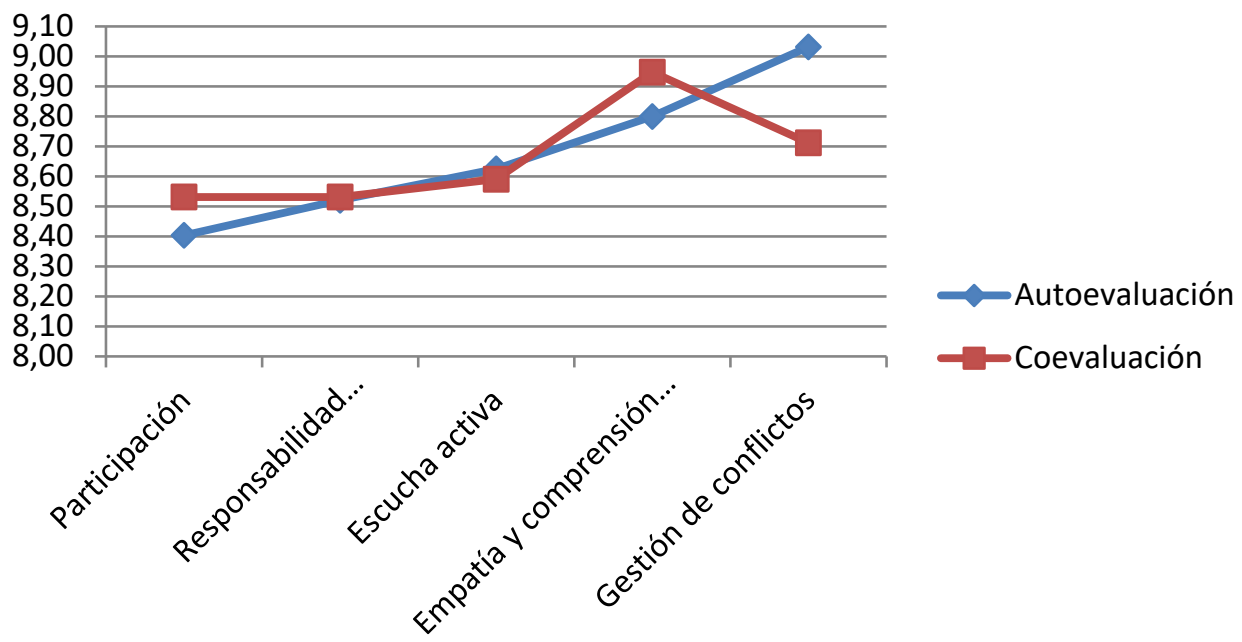

Figura 3: Comparativa de la autoevaluación y la coevaluación para cada dimensión de la rúbrica Fuente: elaboración propia

La figura 3 constata que en la dimensión de “Gestión de conflictos” es donde existe mayor divergencia entre la autoevaluación (con una media de 9 puntos) y la coevaluación (sobre 8,7 puntos). Esto nos aporta insumos para trabajar con el alumnado la empatía hacia el grupo, y el cómo nos posicionamos frente a los conflictos que surgen en los grupos durante el trabajo en equipo.

La figura 4 muestra los indicadores que conforman cada dimensión de la rúbrica. A grandes rasgos se puede ver que las puntuaciones son altas en prácticamente todos los indicadores. 
El trabajo en equipo desde la mirada de los cuidados y la participación: reflexiones de su aplicación en el Máster en Cooperación al Desarrollo por la UPV

Destacan los siguientes indicadores: respeto a las diferencias, compresión de las diferencias y empatía, solidaridad y ambiente pacífico basado en el diálogo. Todas ellas superan el 9.

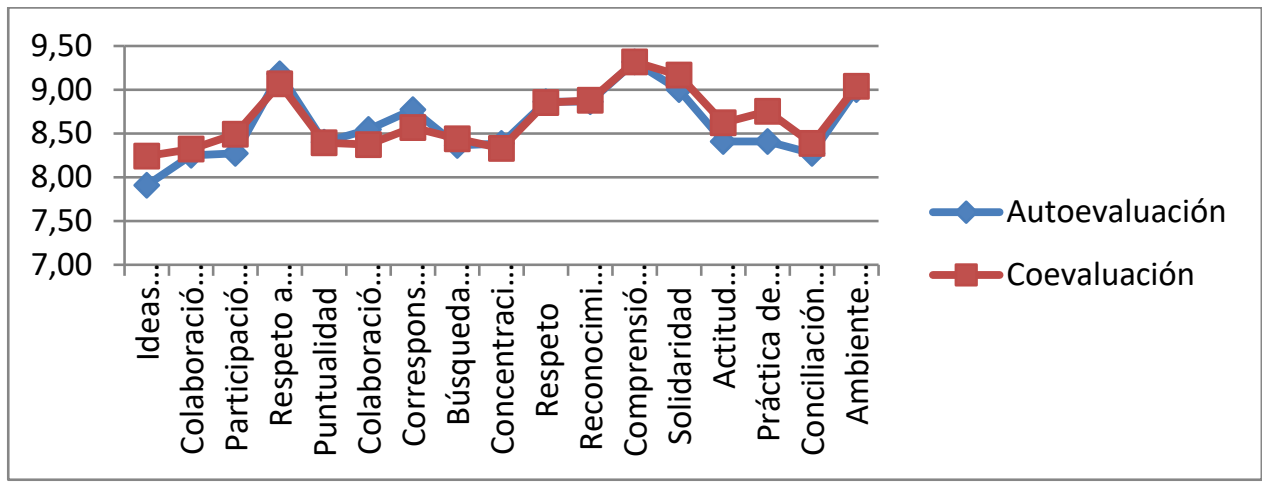

Figura 4: Comparativa de la autoevaluación y la coevaluación para cada indicador de la rúbrica Fuente: elaboración propia

En cambios los indicadores con menor nota son: ideas y voz propia, colaboración y contribución al trabajo en equipo, y asertividad a la hora de escuchar. A la luz de estas cifras son en estos puntos menor valorados donde se puede incidir el año que viene con el alumnado del curso 20182019.

Puesto que una de las novedades de esta rúbrica es la incorporación del enfoque de cuidados, a continuación prestaremos especial atención a este indicador, denominado "Práctica de los cuidados”, dentro de la dimensión Empatía y comprensión mutua.

La Figura 5 muestra el resultado de este indicador para cada estudiante, su autoevaluación, y lo que su grupo opina sobre él.

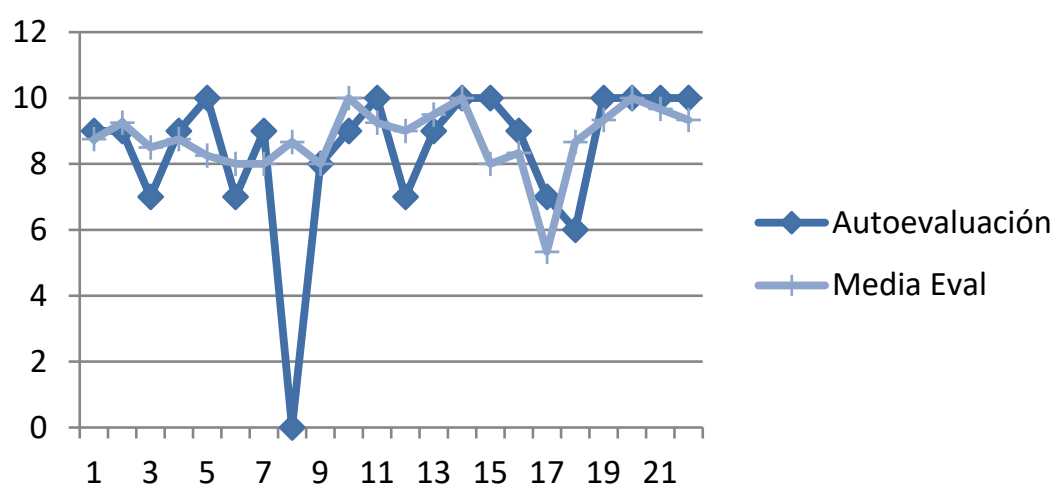

Figura 5: Comparativa de la autoevaluación y la coevaluación para el indicador "Práctica de los cuidados"

Fuente: elaboración propia

Es de destacar que un alumno opina que no practica los cuidados, mientras que la mayoría se autoevalúa con la máxima puntuación. En términos generales destacan las altas puntuaciones,

(cc) EY-NC-ND 2018, Universitat Politècnica de València

Congreso IN-RED (2018) 
tanto de autovaluación, como de coevaluación, lo que nos anima a pensar que introducir el enfoque de cuidados está colaborando a la empatía y comprensión entre cada grupo.

Finalmente, en relación a las prácticas externas, los testimonios hasta la fecha recabados no hacen sino confirmar que la introducción del enfoque de cuidados permite abordar el trabajo en equipo con una mirada necesaria, pero sin perder rigor en el desempeño de una competencia de estas características, como así lo manifiestan las estudiantes: "Creo que el máster ha producido un cambio significativo en la forma en la que las estudiantes hemos aplicado y entendido el trabajo desde los cuidados y la participación. Ha sido algo que ha estado continuamente presente, especialmente el trabajo desde los cuidados, ya que en otros espacios es un tema que se mantiene al margen y para nada forma parte de la pedagogía académica, en cambio, aquí se ha visto reforzado" (estud.5); "considero que las herramientas proporcionadas por el máster en materia de trabajo en equipo desde el enfoque de cuidados y la participación han sido de gran utilidad para las prácticas y son necesarias para el tipo de trabajo al que nos tenemos que enfrentar como futuros cooperantes (estud. 6); "He modificado algunas cosas en mi vida cotidiana y reflexionado sobre mi relación con las personas y mi entorno, gracias a los cuidados y la participación, grandes aprendizajes del máster" (estud. 7). A diferencia de la aplicación de la competencia en el aula, el desempeño del trabajo en equipo en prácticas externas se produce en entornos no académicos, internacionales la mayoría de veces, donde la complejidad y la incertidumbre son mucho mayores. Al ser una iniciativa incipiente, se está trabajando desde el máster para poder conectar mejor el proceso experimentado en el aula con las futuras prácticas, y buscando que el alumnado pueda tener también un seguimiento del trabajo en equipo en la distancia. A la fecha de cierre de la presente comunicación, se están llevando a cabo entrevistas con el alumnado que ha finalizado sus prácticas para evaluarlas, indagar sobre la utilidad del enfoque de cuidados y sistematizar la experiencia.

\subsection{Otros resultados a destacar}

Aunque el presente apartado se dirige a evidenciar los resultados de haber aplicado la competencia transversal bajo los enfoques adoptados, el proceso de elaboración de la rúbrica en sí es un elemento a destacar, dado su carácter participativo. Además, a pesar de que se deben evaluar cinco dimensiones mediante 17 indicadores (lo cual podría parecer farragoso), el alumnado ha valorado muy positivamente este sistema, por cuanto le ha ofrecido descriptores adecuados a diferentes grados de desempeño. La evaluación la han desarrollado como auto y coevaluación (por pares), por lo que era esencial lograr una total comprensión de las rúbricas propuestas.

En relación a los espacios que se han definido para llevar a cabo el seguimiento y lograr al mismo tiempo la reflexión individual y colectiva del alumnado, para el presente curso se ha buscado planificarlos mejor, aunque el desempeño ha sido desigual por cuatrimestres. En el primero, la sesión con el alumnado se produjo antes que en el curso anterior (en el mes de noviembre), permitiendo que los miembros de cada equipo de trabajo de una de las asignaturas dialogaran más entre sí y resolvieran ciertas situaciones problemáticas acontecidas. A pesar de ello, este espacio se presenta insuficiente, por cuanto el trabajo que realizan es no presencial y, por ello, hay una mayor necesidad de verse de forma intencional en el aula. Se sigue evidenciando que el alumnado en el primer cuatrimestre no se conoce todavía bien (acaban de empezar el máster) y 
El trabajo en equipo desde la mirada de los cuidados y la participación: reflexiones de su aplicación en el Máster en Cooperación al Desarrollo por la UPV

que emprender procesos nuevos de golpe les dificulta interiorizar bien lo que están haciendo (por ejemplo, la conexión entre el taller específico en octubre sobre trabajo con cuidados y su propio proceso como equipo según una rúbrica). Las lecciones aprendidas han permitido revisar los mecanismos previstos para el segundo semestre, en el cual el trabajo en aula es mucho más importante. Algunas de las preguntas a las que han respondido individual (de forma anónima) o grupalmente en los espacios para el seguimiento han sido las siguientes: ¿Cómo me estoy sintiendo emocionalmente en mi equipo de trabajo? ¿Qué es lo que más me está preocupando (si ha habido preocupación) de la dinámica de mi grupo durante el proceso? ¿Puedo identificar mi actitud y comportamiento con alguno de estos roles? ¿Qué es lo que más os está preocupando/sorprendiendo positivamente de la dinámica de vuestro equipo durante el proceso?¿Cómo está siendo vuestra comunicación? ¿Qué elementos tratados en el taller sobre trabajo en equipo creéis que habéis podido aplicar en lo que lleváis de trabajo? Cada alumno/a ha sido capaz de reflexionar y responder con sinceridad y, sobre todo, de dialogar en cada equipo a nivel interno.

\section{Conclusiones}

Los resultados obtenidos hasta la fecha, desde abril de 2017, son alentadores, por cuanto la gran mayoría del alumnado considera esencial abordar el trabajo en equipo bajo la mirada de los cuidados y la participación. El hecho de combinar descriptores cualitativos y cuantitativos, ha permitido disponer de diferentes aproximaciones a la valoración que consideramos muy útiles. A pesar de todo ello, se requiere introducir algunas mejoras, asumiendo que cada nueva promoción estará formada por alumnado diverso en cuanto a procedencias geográficas, culturales, formativas y vivenciales, que sin duda plantean un reto año tras año si se quieren tratar cuestiones que se dirigen al ámbito emocional y de las relaciones sociales. En ese sentido, el conflicto acontecido en algunos equipos procede del bajo o limitado desempeño en dimensiones esenciales del trabajo en equipo (más allá del enfoque de cuidados), como es la puntualidad, la responsabilidad y el compromiso sobre las tareas asignadas y en relación al grupo (lograr objetivos previstos y alcanzar resultados óptimos). Al introducir otras dimensiones, relativas al cuidado de la otra persona, a la atención a sus circunstancias personales o al reconocimiento al trabajo del otro/a, la visión sobre el trabajo en equipo cambia y el alumnado reconoce que no solamente es importante "lograr los objetivos marcados de forma eficiente", sino que lo es igualmente o más ser considerados/as sujetos con capacidad de participar y que no pueden eludir sus estados emocionales y personales cuando afrontan una tarea en equipo. El desafío está en cómo trabajar con el alumnado la necesidad de encontrar el equlibrio entre la comprensión del otro/a y la (auto)exigencia y responsabilidad con los compromisos adquiridos.

Finalmente, algunos elementos que deben ser revisados y mejorados serían: la dotación de más espacios presenciales para que los equipos de trabajo dialoguen; aumentar el acompañamiento desde el profesorado; dotar de herramientas para la gestión de conflictos; sistematizar las experiencias como práctica institucionalizada; valorar si es posible extender el enfoque de cuidados a otros ámbitos y propuestas del máster, como el Aprendizaje-Servicio, por ejemplo (InteRed, 2017); profundizar en la colaboración con agentes externos que desde la educación para la ciudadnía global están incorporando el enfoque de cuidados en sus actuaciones. 


\section{Referencias}

BELDA, S. PERIS, J., y CUESTA, I. (2016). "Participation for transformative learning in development management: The case of a Master in international development projects in the Universitat Politècnica de València (Spain)” en Procedia - Social and Behavioral Sciences, 228, p. 237 - 242.

CALABUIG, C., FERNANDEZ-BALDOR, A. y BELDA, S. (2017). "Una nueva mirada a la competencia transversal de trabajo en equipo desde los enfoques de participación y cuidados: El caso del Máster en Cooperación al Desarrollo por la UPV”. Botti , V. y Fernández, M.A.(Eds) En IN-RED 2017. Congreso Nacional de Innovación Educativa y de Docencia en Red.Valencia: Editorial UPV. 867-881.

CARRASCO, C., BORDERÍAS, C., y TORNS, T. (Eds) (2011). El trabajo de los cuidados. Historia, teoría y políticas. Madrid: Los libros de la catarata.

CLARKE, P., y OSWALD, K. (2010). “Why reflect collectively on capacities for change?” en IDS Bulletin, issue 41, p. 1-12.

DE BLAS, A. (2016). La revolución de los cuidados. Tácticas y estrategias. Madrid: InteRed.

EYBEN, R., GUIJT, I., ROCHE, C. y C. SHUTT (Eds.) (2015). The Politics of Evidence and Results in International Development: Playing the game to change the rules?. Rugby: Practical Action Publishing.

GULRAJANI, N. (2010). “New vistas for development management: examining radical-reformist possibilities and potential” en Public Administration and Development, vol. 30, p. 136-148.

HERRERO, Y. (2013). “Miradas ecofeministas para transitar a un mundo justo y sostenible” en Revista de Economía Crítica, nº16, segundo semestre 2013, p. 278-307.

INTERED (2014). La revolución de los cuidados. Tácticas y estrategias. Madrid: InteRed.

INTERED (2017). Guía de aprendizaje servicio con mirada de cuidados. Una aproximación desde nuestras prácticas. Madrid: InteRed.

JOHNSON, H. y THOMAS, A. (2007). "Individual learning and building organizational capacity for development” en Public Administration and Development, vol. 27, p. 39-48.

MCCOURT, W., y GULRAJANI, N. (2010). "The future of development management: Introduction to the Special Issue” en Public Administration and Development, vol.30, p. 81-90.

MOSSE, D. (2005). Cultivating development: An ethnography of aid policy and practice. London: Pluto Press.

ORTIZ ARAGON, A. (2010). "A case for surfacing theories of change for purposeful organizational capacity development” en IDS Bulletin, vol.41, p. 36-46.

PEARSON, J. (2010). "Pushing at a half-open door" en IDS Bulletin, vol. 41, p. 118-127.

PERIS, J., BELDA, S., y CUESTA, I. (2013). "Educating development professionals for reflective and transformative agency” en Boni A. \& Walker M. (Eds.). Human Development and Capabilities: Re-imagining the University of the Twenty-first Century. London and New York: Routledge.

PETTIT, J. (2006). “Power and pedagogy: Learning for reflective development practice” en IDS

Bulletin, vol.37, p. 69-78.

SOAL, S. (2010). “The more things change, the more they stay the same?”, en IDS Bulletin, vol.41, p. 128137. 